\title{
DISPERSION OF SMALL AMPLITUDE STRESS WAVES IN PRE-STRESSED ELASTIC, VISCO-PLASTIC CYLINDRICAL BARS*
}

\author{
BY \\ JOZEF BEJDA AND TOMASZ WIERZBICKI \\ Institute of Basic Technical Research, Polish Academy of Sciences
}

\begin{abstract}
The effect of geometrical dispersion on the propagation of longitudinal harmonic stress waves in prestressed elastic, visco-plastic bars is investigated. The solution involves the Hunter and Johnson approximation in which radial and axial displacements and all components of stress tensor are expanded as power series in the radial coordinate. It is shown that the dispersion of the sinusoidal waves is generated by the two simultaneously acting phenomena. These are the purely geometrical dispersion produced by the influence of a free boundary of the bar and the viscous dispersion caused by the viscous properties of material. The obtained solution embraces the combined effect of the phenomena mentioned above. A numerical example is presented in which constants of material characteristic for mild steel were used. The result of the computations provides a plot of phase and group velocities and damping coefficient against the frequency of harmonic waves. The present solution has been compared with the Hunter and Johnson solution for strain-hardening elastic-plastic material and with the exact Pochhammer-Chree solution for elastic rods.
\end{abstract}

1. Introduction. The theory of wave propagation in elastic/visco-plastic bars has so far been developed on the basis of filament approximation. It was found that the front of longitudinal waves in bars travels always with velocity of corresponding elastic wave $c_{0}$. However the validity of these solutions is restricted to certain loading conditions, since the velocity of the pulse propagated along the bar suffers a frequency-dependent dispersion, which is neglected in the simple theory.

The dispersion of stress waves may be caused by the viscous properties of material. In the case of the Maxwell body the phase velocity increases with wave frequency. When the diameter of the bar is comparable with the wavelength, a dispersion of purely geometrical nature becomes important. For elastic bars the phase velocity decreases with frequency, starting from $c_{0}$, and tends to the velocity of Rayleigh waves. For the material considered here, the two opposing effects mentioned above are present.

The objective of the present paper is to investigate the combined effect of both viscous and geometrical dispersion in elastic/visco-plastic bars and to determine the range of validity of one-dimensional theory. The result of the numerical computations provides a plot of phase velocity and damping coefficient against the frequency of harmonic waves. A similar curve for the group velocity gives some indications concerning the overall pulse velocities and may serve for the proper interpretation of recent experiments on this subject $[1,6]$.

In the present paper, the Hunter and Johnson approximate method [3] is used, in which radial and axial displacements and all components of the stress tensor are expanded as power series in the radial coordinate. Since only small amplitude waves in pre-tensioned bars are studied, the assumption of a linear relaxation function and the

*Received September 30, 1965. 
linearization of the yield condition provides a satisfactorily accurate description of the considered phenomenon. It should be noted that the studies on the effect of finite lateral dimension of a bar within the framework of time-independent plasticity are in progress $[2,3]$. The results of the present paper may be regarded as an attempt at an explanation of the same effect in bars whose material is characterized by more complex mechanical properties.

2. Basic equations. Using a cylindrical coordinate system $r, \varphi, z$, consider an infinite cylindrical bar of the radius $r=a$ and assume that the material of the bar is rate sensitive and can be described by the following constitutive equation (see [5]):

$$
\begin{array}{ll}
\dot{e}_{i j}=\frac{1}{2 \mu} \dot{s}_{i j}+\gamma_{0} \Phi(F) \frac{\partial F}{\partial \sigma_{i j}} & \text { for } \quad \sqrt{J_{2}}>k, \\
\dot{e}_{i j}=\frac{1}{2 \mu} \dot{s}_{i j} & \text { for } \quad \sqrt{J_{2}} \leq k,
\end{array}
$$

where $\dot{e}_{i j}$ and $s_{i j}$ are the components of the strain rate and stress deviators, respectively, $\gamma_{0}, k$ and $\mu$ are material constants, and $J_{2}$ denotes the second invariant of the stress deviation. The sign of $J_{2}-k^{2}$ uniquelly determines whether the behaviour of the material is elastic/visco-plastic or entirely elastic. The visco-plastic flow takes place after the static yield condition has been reached. To ensure that the material is in the elastic, visco-plastic range, we assume that the bar is quasi-statically preloaded to a value $\sigma_{0}^{\prime}$ just above the yield stress $\sigma_{0}$ in pure tension. The corresponding strain rate in the $z$ - direction equals $\dot{\epsilon}_{0}=(2 \gamma / 3 k)\left(\dot{\sigma}_{0}-\sigma_{0}\right)$, while the remaining components of the stress and strain tensors vanish. On this state of stress we superimpose small harmonic disturbances representing an elastic, visco-plastic wave that travels along the bar with the phase velocity $c$. We require the amplitude of the disturbance to be sufficiently small so that no unloading occurs during the considered process. This assumption is of great importance since it allows for considerable simplification of (2.1). Firstly, since all components of stress tensor varies within narrow ranges the linear function $\Phi(F)=F$ would give a satisfactory description of the strain rate sensitivity of the material. Secondly, the nonlinear yield condition $F=\sqrt{J_{2}} / k-1$ can be linearized by expanding the function $\sqrt{J_{2}}$ in Taylor series around the point $P_{0}$ which determines the state of stress prior to the incremental disturbance.

In the case of axial symmetry the components $\tau_{r \varphi}$ and $\tau_{s \varphi}$ vanish and the function $F$ is expressed in terms of remaining components of the stress tensor as

$$
F=\frac{\sqrt{\left(\sigma_{r}-\sigma_{\varphi}\right)^{2}+\left(\sigma_{r}-\sigma_{z}\right)^{2}+\left(\sigma_{\varphi}-\sigma_{z}\right)^{2}+6 \tau_{r z}}}{\sqrt{6} k}-1 .
$$

By expanding (2.2) in Taylor series around the point $P_{0}\left(\sigma_{z}=\sigma_{0}, \sigma_{r}=\sigma_{\varphi}=\tau_{r z}=0\right)$ and neglecting second and higher powers an approximate expression for $F$ is obtained

$$
F=\frac{2 \sigma_{z}-\sigma_{r}-\sigma_{\varphi}}{2 \sigma_{0}}-1 .
$$

The same result was obtained by Hunter and Johnson on the basis of somewhat similar arguments.

It should be stressed here that no methods other than the linearization of (2.1) with respect to the components of stress tensor seems to be possible to treat analytically the considered problem. 
The non-homogeneous terms in (2.1) can be easily eliminated introducing the E. H. Lee substitution [4]

$$
\begin{array}{ll}
\bar{\sigma}_{z}=\sigma_{z}-\sigma_{0}^{\prime}, & \dot{\bar{\epsilon}}_{r}=\dot{\epsilon}_{r}+\frac{1}{2} \dot{\epsilon}_{0}, \\
\dot{\epsilon}_{z}=\dot{\epsilon}_{z}-\dot{\epsilon}_{0}, & \dot{\bar{\epsilon}}_{\varphi}=\dot{\epsilon}_{\varphi}+\frac{1}{2} \dot{\epsilon}_{0} .
\end{array}
$$

Now, using (2.3) and (2.4) we obtain from equation (2.1) four independent equations

$$
\begin{aligned}
& \dot{\bar{\epsilon}}_{z}=\frac{1}{E}\left[\dot{\bar{\sigma}}_{z}-\nu\left(\dot{\sigma}_{r}-\dot{\sigma}_{\varphi}\right)\right]+\frac{\gamma}{3 k}\left[2 \bar{\sigma}_{z}-\left(\sigma_{r}+\sigma_{\varphi}\right)\right], \\
& \dot{\bar{\epsilon}}_{r}=\frac{1}{E}\left[\dot{\sigma}_{r}-\nu\left(\dot{\sigma}_{\varphi}-\dot{\bar{\sigma}}_{z}\right)\right]-\frac{\gamma}{6 k}\left[2 \bar{\sigma}_{z}-\left(\sigma_{r}-\sigma_{\varphi}\right)\right], \\
& \dot{\bar{\epsilon}}_{\varphi}=\frac{1}{E}\left[\dot{\sigma}_{\varphi}-\nu\left(\dot{\bar{\sigma}}_{z}-\dot{\sigma}_{r}\right)\right]-\frac{\gamma}{6 k}\left[2 \bar{\sigma}_{z}-\left(\sigma_{r}-\sigma_{\varphi}\right)\right], \\
& \dot{\gamma}_{r z}=\frac{1+\nu}{E} \dot{\tau}_{r z} .
\end{aligned}
$$

In the cylindrical coordinate system the components of strain rate tensor are related to radial and axial displacements $u_{r}$ and $u_{z}$ by

$$
\dot{\boldsymbol{\epsilon}}_{z}=\frac{\partial^{2} u_{z}}{\partial z \partial t}, \quad \dot{\boldsymbol{\epsilon}}_{r}=\frac{\partial^{2} u_{r}}{\partial r \partial t}, \quad \dot{\boldsymbol{\epsilon}}_{\varphi}=\frac{\partial}{\partial t}\left(\frac{u_{r}}{r}\right), \quad \dot{\gamma}_{r z}=\frac{1}{2}\left[\frac{\partial^{2} u_{r}}{\partial z \partial t}+\frac{\partial^{2} u_{z}}{\partial r \partial t}\right] .
$$

Equations of motion in radial and axial directions have the form

$$
\begin{aligned}
\frac{\partial \sigma_{r}}{\partial r}+\frac{\sigma_{r}-\sigma_{\varphi}}{r}+\frac{\partial \tau_{r z}}{\partial z} & =\rho \frac{\partial^{2} u_{r}}{\partial t^{2}}, \\
\frac{\partial \sigma_{z}}{\partial z}+\frac{\partial \tau_{r z}}{\partial r}+\frac{\tau_{r z}}{r} & =\rho \frac{\partial^{2} u_{z}}{\partial t^{2}} .
\end{aligned}
$$

In the case of infinite cylindrical bar the single boundary condition is the requirement that the components $\sigma_{r}$ and $\tau_{r z}$ vanish on the surface $r=a$

$$
\tau_{r z}(a, t)=\sigma_{r}(a, t)=0 .
$$

Equations (2.5)-(2.7) furnish a system of six equations with six unknown functions (radial and axial displacement and four components of stress tensor). An exact analytical solution of these equations does not seem to be possible. Therefore an effective approximate method developed and successfully used by Hunter and Johnson has been employed to reach the frequency algebraic equations and further results.

3. Solution of the Problem. Following [3] we use the power series expansion method and assume the solution in the form

$$
\begin{aligned}
\bar{u}_{z} & =u_{0}(z, t)+r^{2} u_{2}(z, t), \\
\bar{u}_{r} & =r \omega_{1}(z, t)+r^{3} \omega_{3}(z, t), \\
\sigma_{z} & =h_{0}(z, t)+r^{2} h_{2}(z, t), \\
\sigma_{r} & =f_{0}(z, t)+r^{2} f_{2}(z, t), \\
\sigma_{\varphi} & =f_{0}(z, t)+r^{2} g_{2}(z, t), \\
\tau_{r z} & =r k_{1}(z, t)+r^{3} k_{3}(z, t),
\end{aligned}
$$


preserving the Hunter and Johnson notation. Thus, the problem of determination of six unknowns which are functions of three independent variables $r, z, t$ has been reduced to the solution of eleven equations for eleven functions which depend now upon two arguments $z, t$.

After substitution of (3.1) into equations of flow (2.5), equations of motion (2.7) and boundary conditions (2.8) and equating coefficients of $r^{n}(n=0,1,2)$, the following system is obtained

$$
\begin{aligned}
& (1-\nu) \dot{f}_{0}-\nu \dot{h}_{0}+\alpha f_{0}-\alpha h_{0}=E \dot{\omega}_{1}, \\
& \dot{f}_{2}-\nu \dot{g}_{2}-\nu \dot{h}_{2}-\alpha h_{2}+\frac{\alpha}{2} f_{2}+\frac{\alpha}{2} g_{2}=3 E \dot{\omega}_{3}, \\
& \dot{g}_{2}-\nu \dot{h}_{2}-\nu \dot{j}_{2}-\alpha h_{2}+\frac{\alpha}{2} f_{2}+\frac{\alpha}{2} g_{2}=E \dot{\omega}_{3}, \\
& \dot{h}_{0}-2 \nu f_{0}+2 \alpha h_{0}-2 \alpha f_{0}=E \dot{u}_{0}, \\
& \dot{h}_{2}-\nu \dot{f}_{2}-\nu \dot{g}_{2}-2 \alpha h_{2}-\alpha f_{2}-\alpha g_{2}=E \dot{u}_{2}^{\prime}, \\
& 2(1+\nu) \dot{k}_{1}=2 E \dot{u}_{2}+E \dot{\omega}_{1} \\
& 3 f_{2}-g_{2}+k 1=\rho \ddot{\omega}_{1}, \\
& h_{0}^{\prime}+2 k_{1}=\rho \ddot{u}_{0} \\
& h_{2}^{\prime}+4 k_{3}=\rho \ddot{u}_{2}, \\
& f_{0}+a^{2} f_{2}=0 \\
& k_{1}+a^{2} k_{3}=0 .
\end{aligned}
$$

The prime and dot denote here differentiation with respect to axial distance $z$ and time respectively and parameter $\alpha$ is defined as $\alpha=\gamma E / 3 k$. The validity of the assumption in which terms of order $r^{3}$ and higher are neglected has been extensively discussed by Hunter and Johnson [3]. This method has proved more powerful than many of the previous approximations employed for the elastic problem.

We assume the eigenfunction solution for any one of the functions $u_{0}, u_{2}$, etc.

$$
\varphi_{i}=A_{j} e^{-\eta z} \exp \left[i \omega\left(t-\frac{z}{c(\omega)}\right)\right] \quad(j=1,2, \cdots, 11) .
$$

The amplitude of all functions decay exponentially with axial distance $z$ and the damping coefficient $\eta(\omega)$ is also function of frequency of harmonic waves. Substitution of eigenfunction solution (3.3) into (3.2) yields eleven algebraic equations for eleven unknown amplitudes $A_{1}, A_{2}, \cdots, A_{11}$. This system has a unique solution only when its fundamental determinant is equal to zero

$$
\left|A_{k l}\right|=0 .
$$

The non-vanishing elements of the matrix $A_{k l}$ are

$$
\begin{aligned}
& A_{13}=\frac{1}{3} A_{24}=A_{34}=\frac{1}{2} A_{62}=-E i \omega, \\
& \left.A_{15}=A_{26}=A_{36}=\frac{1}{2} A_{47}=A_{58}=A_{59}=-v i \omega+\alpha\right),
\end{aligned}
$$




$$
\begin{gathered}
A_{17}=(1-\nu) i \omega+\alpha, \quad A_{28}=A_{39}=\frac{\alpha}{2}+i \omega, \quad A_{29}=A_{38}=\frac{\alpha}{2}-\nu i \omega, \\
A_{41}=A_{52}=-A_{63}=E i \omega\left(i \omega+\frac{\eta}{c}\right), \quad A_{45}=A_{56}=2 \alpha+i \omega, \\
A_{6,10}=-2(1+\nu) i \omega, \quad A_{73}=A_{81}=A_{92}=\rho \omega^{2}, \\
A_{7.10}=A_{85}=A_{96}=-\left(\frac{i \omega}{c}+\eta\right) ; \quad \frac{1}{3} A_{78}=-A_{79}=\frac{1}{2} A_{8.10}=\frac{1}{4} A_{9.11}=A_{10.7} \\
=A_{11.10}=1 ; \quad A_{10.8}=A_{11.11}=a^{2} .
\end{gathered}
$$

After rather lengthy, but elementary computations the determinant (3.4) can be evaluated and written in the form

$$
f(\omega, c, \eta)+i \omega g(\omega, c, \eta)=0
$$

which is equivalent to the following system of equations

$$
\begin{aligned}
& f(\omega, c, \eta)=0, \\
& g(\omega, c, \eta)=0 .
\end{aligned}
$$

It is convenient to introduce dimensionless functions and independent variables defined by

$$
x=\frac{a \omega}{c_{0}}, \quad y=a \eta, \quad v=\frac{c}{c_{0}}, \quad \beta=\frac{a \alpha}{c_{0}},
$$

where $c_{0}^{2}=E / \rho$ is elastic velocity in filament approximation. Both functions $f(x, y, v)$ and $g(x, y, v)$ have a polynominal form with coefficients depending solely upon the Poisson ratio $\nu$ and non-dimensional material constant $\beta$ :

$$
\begin{aligned}
& f(x, y, v)=A_{i j} y^{i} v^{j}+x^{2} B_{i j} y^{i} v^{j}+x^{4} C_{i j} y^{i} v^{j}+x^{6} D_{i j} y^{i} v^{j}, \\
& g(x, y, v)=\bar{A}_{i j} y^{i} v^{j}+x^{2} \bar{B}_{i j} y^{i} v^{i}+x^{4} \bar{C}_{i j} y^{i} v^{j} \\
& \quad+x^{6} \bar{D}_{i j} y^{i} v^{j}+x^{8} \bar{E}_{i j} y^{i} v^{i}, \quad(i, j=0,1, \cdots, 6) .
\end{aligned}
$$

The non-vanishing elements of square matrices $A_{i j}, B_{i j}, C_{i i}, D_{i j}, \bar{A}_{i j}, \bar{B}_{i j}, \bar{C}_{i i}$, $\bar{D}_{i i}, \bar{E}_{i j}$ are listed below

$$
\begin{aligned}
A_{26} & =-B_{04}=\frac{1}{2} \bar{B}_{15}=32(5-4 \nu) \beta \\
A_{46} & =-\frac{1}{6} B_{24}=C_{02}=\frac{1}{4} \bar{B}_{35}=-\frac{1}{4} \bar{C}_{13}=8(1+\nu) \beta, \\
\frac{1}{6} A_{55} & =-\frac{1}{20} B_{33}=\frac{1}{6} C_{11}=-\bar{A}_{66}=\frac{1}{15} \bar{B}_{44}=-\frac{1}{15} \bar{C}_{22}=\bar{D}_{00}=\beta^{2}, \\
A_{66} & =-\frac{1}{15} B_{44}=\frac{1}{15} C_{22}=-D_{00}=-\frac{1}{20} \bar{C}_{33}=\frac{1}{8} \bar{D}_{11}=4 \beta, \\
B_{06} & =32\left(9-4 \nu-4 \nu^{2}\right) \beta, \quad \frac{1}{8} B_{15}=\bar{B}_{46}=\bar{D}_{02}=-\left[16\left(1-\nu^{2}\right)+6(2-\nu) \beta^{2}\right], \\
B_{26} & =-C_{04}=\frac{1}{2} \bar{C}_{15}=-48\left(3-2 \nu-2 \nu^{2}\right), \\
B_{35} & =-C_{13}=\frac{2}{3} \bar{C}_{24}=64\left(1-\nu^{2}\right)+24(2-\nu) \beta^{2}, \\
B_{46} & =28-16 \nu-8 \nu^{2}, \quad \frac{1}{6} B_{55}=-\frac{1}{20} C_{33}=\frac{1}{6} D_{11} \\
& =-\bar{B}_{66}=\frac{1}{15} \bar{C}_{44}=-\frac{1}{15} \bar{D}_{22}=\bar{E}_{00}=-(\nu+3)(1-\nu),
\end{aligned}
$$




$$
\begin{aligned}
C_{06} & =-8(1+\nu)\left(25-44 \nu+12 \nu^{2}\right) \beta, \\
\frac{1}{2} C_{15} & =-\bar{C}_{26}=\bar{D}_{04}=8(1+\nu)\left(7-7 \nu-2 \nu^{2}\right)+\left(47-44 \nu-4 \nu^{2}\right) \beta^{2}, \\
\frac{2}{3} C_{24} & =-4 D_{02}=D_{13}=-16\left(7-4 \nu-2 \nu^{2}\right) \beta, \\
C_{26} & =-D_{04}=\frac{1}{2} \bar{D}_{15}=8(1+\nu)(7-11 \nu) \beta, \\
D_{06} & =-\bar{D}_{06}=8(1+\nu)^{2}\left(4-10 \nu+4 \nu^{2}\right) \beta, \\
\frac{1}{4} D_{13} & =\bar{C}_{46}=-\frac{1}{6} \bar{D}_{24}=\bar{E}_{02}=2(1+\nu)\left(6-7 \nu-\nu^{2}\right), \\
\frac{1}{2} D_{15} & =\bar{D}_{26}=\bar{E}_{04}=-(1+\nu)^{2}\left(15-28 \nu+4 \nu^{2}\right), \bar{B}_{06}=-64(5-4 \nu) \beta^{2}, \\
\bar{B}_{26} & =-\bar{C}_{04}=64\left(1-\nu^{2}\right)+24(1-2 \nu) \beta^{2}, \\
\bar{C}_{06} & =64\left(1-\nu^{2}\right)+8\left(31-46 \nu+4 \nu^{2}\right) \beta^{2}, \\
\bar{C}_{35} & =16\left(7-4 \nu-2 \nu^{2}\right), \quad \bar{E}_{06}=2(1+\nu)^{3}(3-2 \nu)(1-2 \nu),
\end{aligned}
$$

Equations (3.9) relate the phase velocity $v(x)$ and damping coefficient $y(x)$ to the frequency of harmonic waves $x$. Explicit expressions for $y$ and $v$ in terms of $x$ could not be found, so that recourse was made to numerical analysis for the determination of dispersion curves.

4. Discussion. It is important to determine an exact asymptotic values for $v$ and $y$ when $x \rightarrow \infty$ and also an expansion at the point $x=0$. For very high frequency waves, $x \rightarrow \infty$ and equations (3.9) yield

$$
\begin{aligned}
& D_{i j} y^{i} v^{i}=0, \\
& \bar{E}_{i j} y^{i} v^{i}=0 .
\end{aligned}
$$

It is easily seen that eq. (4.2) can be solved for $v$ and the value of $y$ can then be computed from (4.1). Equation (4.2) is identical with the corresponding asymptotic frequency equation for elastic waves (see Hunter and Johnson [3]). One of its three real roots has a physical significance and is equal to

$$
v_{1}=[2(1+\nu)]^{-\frac{1}{2}} \text {. }
$$

In the dimensional form the asymptotic velocity is equal to the velocity of distortional waves $c_{1}^{2}=\mu / \rho$ and differs only slightly from the known expression for the velocity of Rayleigh waves. From (4.3) and (4.1) we immediately determine the asymptotic value of damping coefficient which is equal to $y=0$. These results are of great importance since they constitute a proof that the behaviour of elastic, visco-plastic bar at high frequency harmonic wave is purely elastic.

The slope of the curves $v(x)$ and $y(x)$ can be found according to the formulae

$$
\begin{aligned}
& \frac{d v}{d x}=\left(g_{y} f_{x}-f_{y} g_{x}\right)\left(f_{y} g_{v}-g_{y} f_{v}\right)^{-1}, \\
& \frac{d y}{d x}=\left(g_{x} f_{v}-f_{x} g_{v}\right)\left(f_{y} g_{v}-g_{y} f_{v}\right)^{-1},
\end{aligned}
$$

where indices $x, y$, and $v$ denote partial differentiation.

On the other hand for $x \rightarrow 0$ (3.9) reduce to

$$
A_{i j} y^{i} v^{i}=0, \quad \bar{A}_{i j} y^{i} v^{i}=0 .
$$

It is found that $\lim _{x \rightarrow 0} v(x)=0$ and $\lim _{x \rightarrow 0} y(x)=0$, whereas the first derivative $d v / d x$ and $d y / d x$ tend to infinity. This behaviour corresponds to the viscous dispersion for the 
Maxwell model. The zero phase velocity for $x=0$ is explained by the fact that there is no mechanism to transmit an infinitely long wave in the pre-stressed bar.

In the experiments on propagation of waves in bars the direct measurable quantity is group velocity $v_{o}=c_{o} / c_{0}$ rather than phase velocity $v$. The group velocity can be found through the differentiation of function $v(x)$, namely

$$
v_{s}(x)=v(x)\left[1-\frac{x}{v} \frac{d v}{d x}\right]^{-1}
$$

On account of formula (4.4) we have the following expression for the group velocity

$$
v_{g}(x)=v(x)\left[1-\frac{x}{v}\left(f_{y} g_{v}-g_{y} f_{v}\right)^{-1}\left(g_{y} f_{x}-f_{v} g_{x}\right)\right]^{-1} .
$$

(4.8) enables one to compute $v_{0}$ without a graphical differentiation of dispersion curve which generally is not sufficiently accurate.

The constitutive equations (2.1) turn in the limiting case $\gamma_{0} \rightarrow 0$ into the corresponding relation describing the purely elastic material. The amplitude of elastic waves suffers no damping and therefore we can assume $\eta=y=0$. By introducing $y=\beta=0$ into the equations (3.9) it appears that the first equation (3.9) is satisfied identically whereas the second equation yields an algebraic frequency equation derived earlier by Hunter and Johnson (eq. (2.14) of the paper [3]). It is evident that for vanishingly small viscosity constant $\beta$ the curve $y(x)$ would tend to the known Pochhammer-Chree dispersion curve.

5. Numerical Example. We consider a mild steel cylindrical bar of the radius $a=1 \mathrm{~cm}$. Coefficients in the frequency equations depend upon the Poisson ratio $\nu$ and constant $\beta$ defined by (3.8). To compute $\beta$ we assume the following values for material constants representative for mild steel (Table 1). The system of equations (3.9) has been solved numerically on the GIER electronic computer in the range $0 \leq x \leq 10$, and the results are plotted in Figs. 1 and 2. On the same figures dispersion curves corresponding to

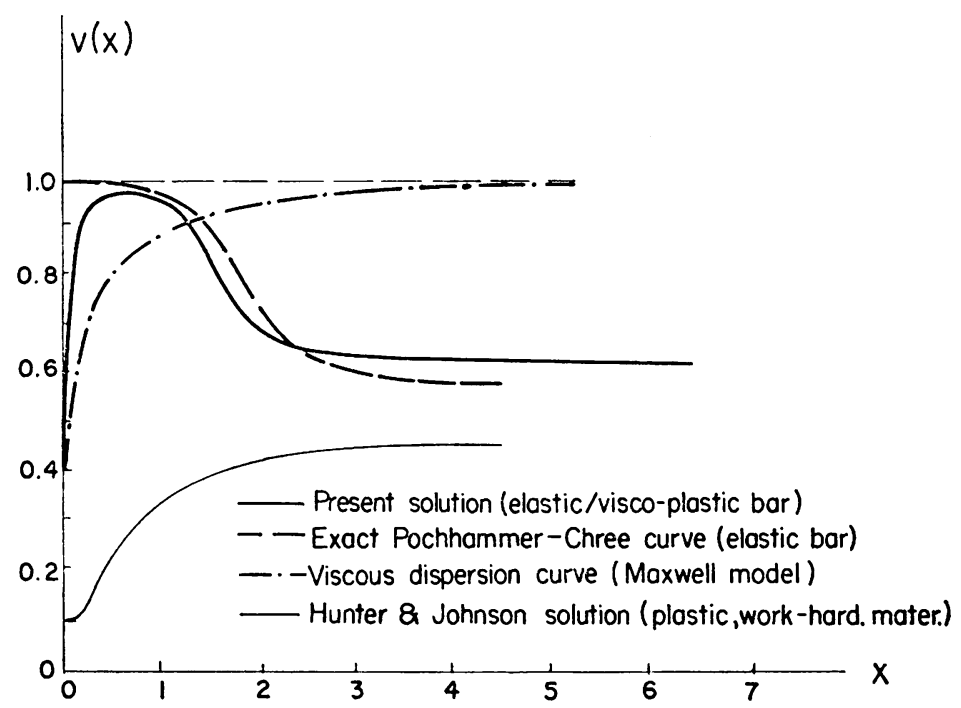

FIg. 1. Phase velocity against frequency of harmonic waves. 
TABLE 1

\begin{tabular}{|c|c|c|c|c|c|c|}
\hline$E$ & $\rho$ & $k$ & $\nu$ & $\gamma$ & $\beta$ & RN \\
\hline $2,1 \cdot 10^{6}$ & $7,8 \cdot 10^{-6}$ & $2 \cdot 10^{3}$ & 0,3 & 148 & 0,1 & RN \\
\hline $\mathrm{KG} \mathrm{\textrm {cm } ^ { - 2 }}$ & $\mathrm{KG} \mathrm{cm}^{-4} \mathrm{sec}^{2}$ & $\mathrm{KG} \mathrm{cm}^{-2}$ & - & $\sec ^{-1}$ & - & R.N \\
\hline
\end{tabular}

purely geometrical and purely viscous dispersion are also shown. It is seen that the curve $v(x)$ reaches its maximum near $x=0.75$, where the phase velocity differs by a few percent from the value predicted by the one-dimensional theory. The results of computations indicate that for very low frequencies the effect of viscous dispersion is predominant. Starting from the value $x \cong 0.5$ the curve $v(x)$ approaches the dispersion curve of Pochammer-Chree.

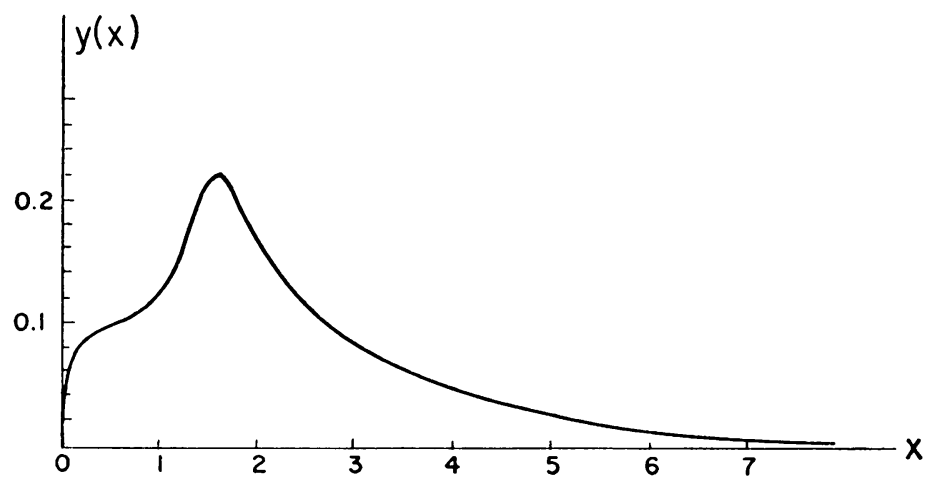

FIg. 2. Damping coefficient against frequency of harmonic waves.

This means that within the range of high frequencies the purely geometrical dispersion becomes more significant.

The plot of group velocity $v_{0}$ against frequency, computed from (4.8) is shown in Fig. 3. It is seen that $v_{o}$ nowhere exceeds the value $v_{o}=1$ except the very low frequencies $x \rightarrow 0$. This result would give a theoretical explanation of Sternglass and Stuart experiments on copper strips [6] where the wave fronts of pulses were observed to travel at the elastic velocity $c_{0}$ whereas the overall pulse velocities were slightly smaller.

\section{ReFERENCES}

1. G. Bianchi, Some experimental and theoretical studies on the propagation of longitudinal plastic waves in a strain-rate-dependent materials, Stress Waves in Anelastic Solids, Proc. IUTAM Symposium, Providence 1963, 101-117, Springer (1964)

2. G. P. De Vault, The effect of lateral inertia on the propagation of plastic strain in a cylindrical rod, J. Mech. Phys. Solids 13, 55-68 (1965)

3. S. C. Hunter and I. A. Johnson, The propagation of small amplitude elastic-plastic waves in pre-stressed cylindrical bars, Stress Waves in Anelastic Solids, Proc. IUTAM Symposium, Providence 1963, 149-165, Springer (1964)

4. E. H. Lee, Wave propagation in anelastic materials, Deformation and Flow of Solids, IUTAM Colloquium, Madrid 1955, 129-136, Springer (1956) 


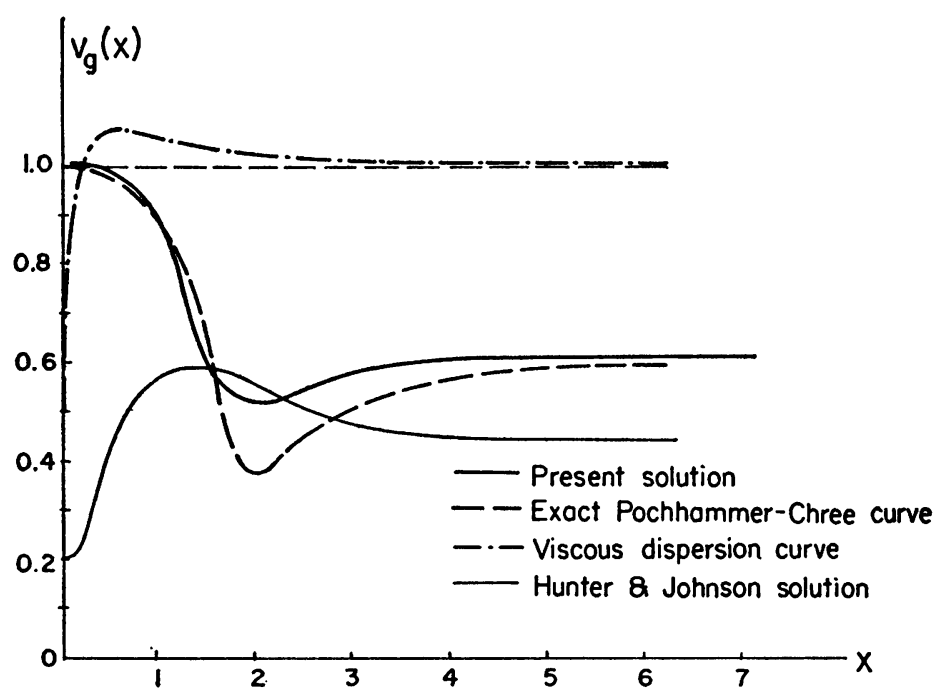

FIG. 3. Group velocity against frequency of harmonic waves.

5. P. Perzyna, The constitutive equations for rate sensitive plastic materials, Quart. Appl. Math., 20, 321-332 (1963)

6. E. J. Sternglass and D. A. Stuart, An experimental study of the propagation of transient longitudinal deformation in elasto-plastic media, J. Appl. Mech., 20, 427 (1953) 